\title{
Studies on Fertility Restoration Using Newly Derived Restorers in Sunflower (Helianthus annuus L.)
}

\author{
H.A. Bhargavi ${ }^{1^{*}}$ and A.G. Vijayakumar ${ }^{2}$ \\ ${ }^{1}$ Department of Genetics and Plant Breeding, college of agriculture, \\ Vijayapur, Karnataka, India \\ ${ }^{2}$ AICRP for Dryland Agriculture, RARS, Vijayapur, Karnataka, India \\ *Corresponding author
}

\begin{abstract}
Keywords
Sunflower,

Restorers, Fertility

restoration

Article Info

Accepted:

16 December 2017

Available Online:

10 January 2018
\end{abstract}

\section{A B S T R A C T}

Improvement in sunflower emphasizes the urgency of generating heterotic hybrids that is achieved by tapping the excellent combining ability and heterotic vigour available in the genetically divergent material. Concomitant with major diseases prevailing largely and most of the sunflower experimental hybrids and cultivars being based on single cytoplasm, large scale cultivation of the crop finds itself in greater threat as evident by susceptibility tonew emerging diseases and declining yield levels over the decades. However, efforts are being made to diversify both cytoplasm and the restorers, but with very limited success. To augment resistance breeding and improve yield levels, fertility restoration in three different CMS lines of sunflower was studied using sixteen recombinant inbred lines derived from an exotic line TX 16Rthrough introgression breeding as well as chemical mutagenesis. The new inbreds behaved as either maintainer/restorer on different CMS lines. Among the sixteen new inbreds tested, eight inbreds (DSR-121, DSR-122, DSR-124, DSR-128, DSR131, DSR-132, DSR-134 and DSR-136) behaved as restorers, while the inbred DSR-125 behaved as a maintainer on CMS 103A. However, none of the restorer lines completely restored fertility on the female lines CMS-302A and CMS-PET27A. The study identified several effective restorers derived from an exotic line TX16R, which can be utilized in production of highly heterotic and resistant sunflower hybrids.

\section{Introduction}

Hybrid breeding has developed successfully in sunflower over the last 30 years since the identification of cytoplasmic male sterility among progenies of the interspecific cross Helianthus petiolaris $\mathrm{x}$ Helianthus annuиs by Leclercq (1969) and the subsequent discovery of pollen fertility restoration genes (Kinman, 1970; Leclercq, 1971; Vranceanu and Stoenescu, 1971). This source (PET 1 cytoplasm), of cytoplasmic male sterility has proved to be very stable and is used almost exclusively in breeding programmes throughout the world since late 1970s, when it replaced the NMS system for producing hybrid seeds that was being used in several European countries till the early 1970s.Since then, many hybrids have been released for commercial cultivation by utilizing cytoplasmic genetic male sterility system. The 
lack of new CMS and fertility restoration (Rf) genes for commercial hybrid production is fairly due to the limited number of stable cytoplasmic sources and their corresponding fertility restorer genes. Further, the conversion of potential lines to CMS sources is time consuming and laborious as is the process of incorporation of new $\mathrm{Rf}$ genes into adapted cultivated lines inviting attention of few breeder and thereby limiting the potential utility of sunflower hybrids. Hence, it is necessary to assess the role of new inbreds for fertility restoration as well as their effect on seed yield and oil content.

Success in heterosis breeding is largely dependent on the development of inbreds having broader genetic base. In general, inbreds with high combining ability and per se performance are either converted into CMS lines or fertility restorer lines for their future use in hybrid breeding. Besides developing new sources of male sterility, identification of new restorer lines will enhance the genetic diversity. TX-16 R, a USDA germplasm behaves as a restorer, but has few undesirable characters, limiting its direct utility in hybrid programme. In present study thirteen new recombinant inbred lines DSR-122, DSR-123, DSR-124， DSR-125， DSR-126， DSR-127, DSR-129， DSR-130， DSR-131， DSR-132, DSR-133, DSR-135 and DSR-136 were derived from [(CMS 4546 x DSF-2A) x TX $16 \mathrm{R}]$ and three mutant inbreds (DSR-121, DSR-128 and DSR- 134)through induced mutagenesis (EMS treatment of TX 16R seeds at 0.020 moles $/ \mathrm{dm} 3)$. A total of 48 experimental hybrids were produced from crossing 3 cytoplasmic male sterile lines with the sixteen newly derived recombinant/ mutant inbreds in line $\mathrm{x}$ tester mating design during summer 2015. The hybrids were evaluated during late kharif 2015 at Regional Agricultural Research Station, Vijayapur in a randomized block design with three replications. Identification of selected superior inbreds as maintainers, partial restorers and restorers on three different CMS sources, for further utility in sunflower breeding programme to augment the genetic diversity of sunflower hybrids was done.

\section{Materials and Methods}

Three different cytoplasmic male sterile lines viz., CMS-302A, CMS-103A and CMSPET27Awere crossed with the newly derived inbreds in line $\mathrm{x}$ tester design to derive 48 hybrids during summer 2015 at Regional Agricultural Research station, Vijayapur. $F_{1}$ seeds from each of the forty eight crosses were collected separately at maturity for assessing the fertility restoration. The identification of inbred behaviour, with respect to maintenance or fertility restoration was conducted during the latekharif2015 at Regional agricultural research station, Vijayapur. Hybrid seeds from the 48 crosses were planted in a replicated experiment with three replications. For fertility restoration studies, the number of plants with or without pollen shedding in each treatment was recorded to work out per cent fertility. Based on these observations, the crosses were grouped as either sterile or fertile. The pollen parents leading to sterile crosses were classified as maintainers, while those that gave fertile crosses were classified as restorers of the corresponding CMS lines.

\section{Results and Discussion}

Sixteen different new restorer lines evaluated turnout to be either partial restorers on different CMS backgrounds or complete restorers. New restorer lines DSR-121, DSR122, DSR-124, DSR-128, DSR-131, DSR132, DSR-134 and DSR-136 restored complete fertility on CMS-103A background (Table 1). Most of the new restorer lines tested behaved as partial restorers. 
Table.1 Fertility restoration behaviour of new restorer lines on three CMS lines of sunflower

\begin{tabular}{|c|c|c|c|c|}
\hline $\begin{array}{l}\text { Sl. } \\
\text { No. }\end{array}$ & Crosses & Per cent fertility & $\begin{array}{l}\text { Status of male } \\
\text { parent fertility } \\
\text { restoration }\end{array}$ & $\begin{array}{c}\text { Status of } \\
\text { hybrid }\end{array}$ \\
\hline 1 & CMS-302A x DSR-121 & 25 & Partial Restorer & $\mathrm{S} / \mathrm{F}$ \\
\hline 2 & CMS-302A x DSR-122 & 55.77 & Partial Restorer & $\mathrm{F} / \mathrm{S}$ \\
\hline 3 & CMS-302A x DSR-123 & 0 & Maintainer & $\mathrm{S}$ \\
\hline 4 & CMS-302A x DSR-124 & 63.46 & Partial Restorer & $\mathrm{F} / \mathrm{S}$ \\
\hline 5 & CMS-302A x DSR-125 & 63.46 & Partial Restorer & $\mathrm{F} / \mathrm{S}$ \\
\hline 6 & CMS-302A x DSR-126 & 76.92 & Partial Restorer & $\mathrm{F} / \mathrm{S}$ \\
\hline 7 & CMS-302A x DSR-127 & 0 & Maintainer & $\mathrm{S}$ \\
\hline 8 & CMS-302A x DSR-128 & 75.00 & Partial Restorer & $\mathrm{F} / \mathrm{S}$ \\
\hline 9 & CMS-302A x DSR-129 & 90.38 & Partial Restorer & $\mathrm{F} / \mathrm{S}$ \\
\hline 10 & CMS-302A x DSR-130 & 36.53 & Partial Restorer & $\mathrm{S} / \mathrm{F}$ \\
\hline 11 & CMS-302A x DSR-131 & 7.69 & Partial Restorer & $\mathrm{S} / \mathrm{F}$ \\
\hline 12 & CMS-302A x DSR-132 & 0 & Maintainer & $\mathrm{S}$ \\
\hline 13 & CMS-302A x DSR-133 & 0 & Maintainer & S \\
\hline 14 & CMS-302A x DSR-134 & 15.38 & Partial Restorer & $\mathrm{S} / \mathrm{F}$ \\
\hline 15 & CMS-302A x DSR-135 & 63.46 & Partial Restorer & $\mathrm{F} / \mathrm{S}$ \\
\hline 16 & CMS-302A x DSR-136 & 38.46 & Partial Restorer & $\mathrm{S} / \mathrm{F}$ \\
\hline 17 & CMS-103A x DSR-121 & 100 & Restorer & $\mathrm{F}$ \\
\hline 18 & CMS-103A x DSR-122 & 100 & Restorer & $\mathrm{F}$ \\
\hline 19 & CMS-103A x DSR-123 & 57.69 & Partial Restorer & $\mathrm{F} / \mathrm{S}$ \\
\hline 20 & CMS-103A x DSR-124 & 100 & Restorer & $\mathrm{F}$ \\
\hline 21 & CMS-103A x DSR-125 & 0 & Maintainer & S \\
\hline 22 & CMS-103A x DSR-126 & 61.53 & Partial Restorer & $\mathrm{F} / \mathrm{S}$ \\
\hline 23 & CMS-103A x DSR-127 & 71.15 & Partial Restorer & $\mathrm{F} / \mathrm{S}$ \\
\hline 24 & CMS-103A x DSR-128 & 100 & Restorer & $\mathrm{F}$ \\
\hline 25 & CMS-103A x DSR-129 & 65.61 & Partial Restorer & $\mathrm{F} / \mathrm{S}$ \\
\hline 26 & CMS-103A x DSR-130 & 76.92 & Partial Restorer & $\mathrm{F} / \mathrm{S}$ \\
\hline 27 & CMS-103A x DSR-131 & 100 & Restorer & $\mathrm{F}$ \\
\hline 28 & CMS-103A x DSR-132 & 100 & Restorer & $\mathrm{F}$ \\
\hline 29 & CMS-103A x DSR-133 & 57.69 & Partial Restorer & $\mathrm{F} / \mathrm{S}$ \\
\hline 30 & CMS-103A x DSR-134 & 100 & Restorer & $\mathrm{F}$ \\
\hline 31 & CMS-103A x DSR-135 & 61.54 & Partial Restorer & $\mathrm{F} / \mathrm{S}$ \\
\hline 32 & CMS-103A x DSR-136 & 100 & Restorer & $\mathrm{F}$ \\
\hline 33 & CMS-PET27A x DSR-121 & 25 & Partial Restorer & $\mathrm{S} / \mathrm{F}$ \\
\hline 34 & CMS-PET27A x DSR-122 & 0 & Maintainer & $S$ \\
\hline 35 & CMS-PET27A x DSR-123 & 0 & Maintainer & S \\
\hline 36 & CMS-PET27A x DSR-124 & 46.15 & Partial Restorer & $\mathrm{S} / \mathrm{F}$ \\
\hline 37 & CMS-PET27A x DSR-125 & 11.53 & Partial Restorer & $\mathrm{S} / \mathrm{F}$ \\
\hline 38 & CMS-PET27A x DSR-126 & 0 & Maintainer & S \\
\hline 39 & CMS-PET27A x DSR-127 & 0 & Maintainer & S \\
\hline 40 & CMS-PET27A x DSR-128 & 0 & Maintainer & S \\
\hline 41 & CMS-PET27A x DSR-129 & 65.38 & Partial Restorer & $\mathrm{F} / \mathrm{S}$ \\
\hline 42 & CMS-PET27A x DSR-130 & 0 & Maintainer & $\mathrm{S}$ \\
\hline 43 & CMS-PET27A x DSR-131 & 0 & Maintainer & $\mathrm{S}$ \\
\hline 44 & CMS-PET27A x DSR-132 & 25 & Partial Restorer & $\mathrm{S} / \mathrm{F}$ \\
\hline 45 & CMS-PET27A x DSR-133 & 0 & Maintainer & S \\
\hline 46 & CMS-PET27A x DSR-134 & 32.69 & Partial Restorer & $\mathrm{S} / \mathrm{F}$ \\
\hline 47 & CMS-PET27A x DSR-135 & 0 & Maintainer & $\mathrm{S}$ \\
\hline 48 & CMS-PET27A x DSR-136 & 36.54 & Partial Restorer & $\mathrm{S} / \mathrm{F}$ \\
\hline
\end{tabular}

F - Fertile $S$ - Sterile 
Table.2 Pattern of fertility restoration by new restorer lines on three CMS lines of sunflower

\begin{tabular}{|c|c|c|c|c|}
\hline Sl. No. & CMS lines & Maintainers & Partial restores & Restorers \\
\hline 1 & CMS-302A & $\begin{array}{l}\text { DSR-123, DSR-127, } \\
\text { DSR-132, DSR-133 }\end{array}$ & $\begin{array}{c}\text { DSR-121, DSR-122, DSR-124, DSR } \\
\text { 125,DSR-126, DSR-128, DSR-129, } \\
\text { DSR-130, DSR-131, DSR-134, DSR- } \\
\text { 135, DSR-136 }\end{array}$ & None \\
\hline 2 & CMS-103A & DSR-125 & $\begin{array}{l}\text { DSR-123, DSR-126, DSR-127, DSR- } \\
\text { 129, DSR-130,DSR-133, DSR-135 }\end{array}$ & $\begin{array}{c}\text { DSR-121, DSR-122, DSR- } \\
\text { 124, DSR-128,DSR-131, } \\
\text { DSR-132, DSR-134, DSR- } \\
136\end{array}$ \\
\hline 3 & CMS-PET 27A & $\begin{array}{l}\text { DSR-122, DSR-123, } \\
\text { DSR-126, DSR-127, } \\
\text { DSR-128, DSR-130, } \\
\text { DSR-131, DSR-133, } \\
\text { DSR-135 }\end{array}$ & $\begin{array}{l}\text { DSR-121, DSR-124, DSR-125, DSR- } \\
\text { 129,DSR-132, DSR-134, DSR-136 }\end{array}$ & None \\
\hline
\end{tabular}

The differential behaviour of new restorer lines developed on different CMS sources indicated significant diversity among new restorers tested for fertility restoration as reported by earlier studies (Divya, 2010; Reddy, 2012; Shakuntala, 2014; Spoorthi, 2015). Among these, sixteen new restorer lines DSR-121, DSR-122, DSR-124, DSR128, DSR-131, DSR-132, DSR-134 and DSR136 behaved as specific combiners for CMS$103 \mathrm{~A}$ with respect to fertility restoration behaviour.

The fertility on CMS-302A was found to be partially restored by DSR-121, DSR-122, DSR-124, DSR 125, DSR-126, DSR-128, DSR-129， DSR-130， DSR-131， DSR-134, DSR-135, and DSR-136, whereas DSR-123, DSR-127, DSR-132, DSR-133 acted as maintainers with 100 per cent sterility, while none of the restorer lines completely restored fertility on this CMS line.

The fertility on CMS-PET27A was found to be partially restored by DSR-121, DSR-124, DSR-125, DSR-129, DSR-132, DSR-134 and DSR-136, whereas DSR-122, DSR-123, DSR-126，DSR-127， DSR-128， DSR-130, DSR-131, DSR-133, DSR-135 were complete maintainers with 100 per cent sterility but none of the male lines completely restored fertility on this CMS line.
One of the new restorer line DSR-129 recorded partial restoration of fertility with all the CMS lines viz., CMS-302A, CMS-103A and CMS-PET27A. Restorer, DSR-129 restored more than 90 per cent fertility on CMS-302A, 65 per cent fertility on CMS103A and CMS-PET27A (Table 1 and 2). Thus, these lines have to be improved further for fertility restoration. The differential behaviour of these new restorers on different CMS lines could be due to variable expression of restoration genes in different environment, their wide genetic diversity as these new restorer lines have been obtained by hybridization on background of different maintainer and restorer lines as well as induced mutagenesis of TX-16R. The exact genetic basis of partial restoration is still unclear, but can be rectified with few more inbreeding cycles to obtain stable inbreds. It may be governed by multiple genes, which may have an effect in the absence of fully restoring genes (Tracy et. al., 1991; Has, 2002). Partial restoration can be overcome by selection of restoration gene, through molecular approach, in a way that restoration is governed by only one fully restoration gene, which is absent in the maternal gene pool.

Nevertheless, efforts toward identification of different restorers for CMS-103A are 
desirable for greater genetic diversity to be used in the development of new restorer inbred lines (Gimenez and Fick, 1975) and the hybrids. The different CMS lines and their concerned maintainer and restorer inbreds of sunflower can be utilized directly in maintenance breeding and hybrid development programme.

\section{References}

Divya, A., 2010. Genetic analysis of diverse sources of CMS on fertility restoration, heterosis and combining ability in sunflower (Helianthus annuus L.).M. Sc. (Agri.) Thesis, University of Agricultural Sciences, Dharwad, Karnataka (India).

Gimenez, D.J., and Fick, G. 1975. Fertility restoration of male sterile cytoplasm in wild sunflower. Crop Science.15: 724726.

Has, V., 2002. Evaluation of some maize inbred lines on fertility restoration patterns of male-sterile cytoplasms. Romanian Agriculture Research.17:1-7.

Kinman, M.L., 1970. New developments in the USDA and state experiment station sunflower breeding programmes. Proceedings of the 4th International Sunflower Conference, June 23-25, 1970, Memphis TN., USA.181-183.

Leclercq, P., 1969. Unesterilite male cytoplasmique chez le tournesol. Ann. Amelior. Plant.19: 99-106.
Leclercq, P., 1971. Lasterilite male cytoplasmique de tournesol. I. Premieres etudes sur la restoration de la fertilite. Ann. Amelior. Plant. 21: 4554.

Reddy P, V. R., 2012. Genetic analysis of seed yield and its component traits and resistance to Alternaria bright in sunflower (Helianthus annuus L.).M. Sc. (Agri.) Thesis, University of Agricultural Sciences, Dharwad, Karnataka (India).

Shakuntala, M. H., 2014. Combining ability analysis of new restorer developed for resistance to Alternaria leaf blight disease in sunflower (Helianthus annuus L.). M. Sc. (Agri.) Thesis, University of Agricultural Sciences, Dharwad, Karnataka (India).

Spoorthi, V., 2015. Heterosis and combining ability analysis of new restorers developed for resistance to alternaria leaf blight disease in sunflower (Helianthus annuus L.).M. Sc. (Agri.) Thesis, University of Agricultural Sciences, Dharwad, Karnataka (India).

Tracy, W. F., Everett, H. L. and Gracen, V. E. 1991. Inheritance, environmental effects, and partial male-fertility in Ctype CMS in a maize inbred. Journal of Heredity. 82: 343-346.

Vranceanu, A.V. and Stoenescu, F.M. 1971. Pollen fertility restorer gene from cultivated sunflower (Helianthus annuus L.). Euphytica. 20: 536-541.

\section{How to cite this article:}

Bhargavi, H.A. and Vijayakumar, A.G. 2018. Studies on Fertility Restoration Using Newly Derived Restorers in Sunflower (Helianthus annuus L.). Int.J.Curr.Microbiol.App.Sci. 7(01): 2131-2135. doi: https://doi.org/10.20546/ijcmas.2018.701.257 